\title{
Nonintegrability of harmonic functions in a domain
}

\author{
By Noriaki SuzukI \\ (Received February 9, 1989)
}

§1. Let $D$ be a domain in the Euclidean space $\boldsymbol{R}^{n}$ with $n \geq 2$. We define a function $\delta_{D}$ in $D$ by

$$
\delta_{D}(x)=\operatorname{dist}(x, \partial D)
$$

and for $p>0$ we put

$$
\alpha(p)= \begin{cases}1 & \text { if } 0<p \leq(n-2) /(n-1) \\ (n-1)(1-p) & \text { if }(n-2) /(n-1)<p \leq 1 \\ 0 & \text { if } p>1\end{cases}
$$

In contrast to [5], our concern in this paper is the nonintegrability of harmonic functions. We state our result in the following form:

Theorem A. Let $D$ be a bounded domain in $\boldsymbol{R}^{n}$ with the exterior sphere property. Assume in addition that there is a countable closed set F in $\partial D$ su ch that $D$ has the locally uniform exterior sphere property on $\partial D \backslash F$ and that $D$ satisfies the Lipschitz condition at every point in $\partial D \backslash F$. If a non-negative harmonic function $h$ in $D$ satisfies

$$
\int_{D} \frac{h(x)^{p}}{\delta_{D}(x)^{1+\alpha(p)}} d x<\infty
$$

with some $p>0$, then $h \equiv 0$ in $D$.

Here, we recall some definitions; we say that $D$ has the exterior sphere property if for each boundary points $\xi \in \partial D$, there exists a ball $B_{\xi}$ such that $B_{\xi} \subset \bar{D}^{c}$ and $\bar{B}_{\xi} \cap \partial D=\{\xi\}$. Further, for an open set $E$ in $\partial D$, if the radius of $B_{\xi}$ can be taken uniformly on any compact set in $E$, we say that $D$ has the locally uniform exterior sphere property on $E$; in case $E=\partial D$ we say that $D$ has the uniform exterior sphere property. Again, let $\xi$ be a boundary point of $D$. We say that $D$ satisfies the Lipschitz condition at $\xi$ if there is a neighbourhood $N$ of $\xi$ such that $D \cap N$ is a Lipschitz domain.

The proof of Theorem $A$ is carried out in Section 5 by combining three theorems below. Indeed, each result is interesting in its own right. The 
first result, which is discussed in Section 2, concerns the nonintegrability of positive superharmonic functions in arbitrary domains. On the other hand, in Section 3, we deal with the nonintegrability of subharmonic functions in $C^{1,1}$-domains. Finally, in Section 4, we study the non-tangential positiveness of positive harmonic functions in certain Lipschitz domains. In particular, as the simplest case, we obtain the following result:

Let $h$ be a non-negative harmonic function in the unit ball $B$ in $\boldsymbol{R}^{n}$. If $\liminf _{r \rightarrow 1} h(r \xi)=0$ for every boundary point $\xi \in \partial B$, then $h$ vanishes identically.

\section{§2. Nonintegrability of superharmonic functions}

Throughout this paper we denote by $B(x, r)$ the open ball of radius $r>0$ centred at $x \in \boldsymbol{R}^{n}$ and by $G_{D}$ the Green function of a domain $D$. We use $c(p, q, \cdots)$ to denote positive constants depending only on $p, q, \cdots$ not necessarily the same on any two occurrences.

We have the following theorem.

THeOREM 1. Let $D$ be a bounded domain in $\boldsymbol{R}^{n}$. If a non-negative superharmonic function $u$ in $D$ satisfies

$$
\int_{D} \frac{u(x)}{\delta_{D}(x)^{2}} d x<\infty
$$

then $u \equiv 0$ in $D$.

Proof. We consider

$$
v(y)=\int_{D} G_{D}(y, x) \delta_{D}(x)^{-2} d x .
$$

For $y \in D$, put $B=B\left(y, \delta_{D}(y)\right)$ and $B^{\prime}=B\left(y, \delta_{D}(y) / 2\right)$. Since $G_{D}(x, y) \geq$ $G_{B}(x, y) \geq c(n)|x-y|^{-n+2}$ for $x \in B^{\prime}$, we have

$$
v(y) \geq c(n) \delta_{D}(y)^{-2} \int_{B^{\prime}}|x-y|^{-n+2} d x \geq c(n)>0 .
$$

This implies $v \equiv \infty$; for, otherwise $v$ is a potential which contradicts the fact that $v$ has a positive harmonic minorant. Hence, for any potential $p=$ $\int G_{D}(\cdot, y) d \nu(y)$ with $\nu \neq 0$, we also see

$$
\int_{D} p(x) \delta_{D}(x)^{-2} d x=\int_{D}\left(\int_{D} G_{D}(x, y) \delta_{D}(x)^{-2} d x\right) d \nu(y)=\infty .
$$

Since every positive superharmonic function has a non-zero potential as its 
minorant, the assertion of Theorem 1 follows as an easy consequence.

\section{Nonintegrability of subharmonic functions}

For $p>0$ we put $\beta(p)=\max \{(n-1)(1-p), 0\}$. We have the following theorem.

Theorem 2. Let $D$ be a bounded $C^{1,1}$-domain in $R^{n}$ and $p>0$. If $a$ non-negative subharmonic function $s$ in $D$ satisfies

$$
\int_{D} \frac{s(x)^{p}}{\delta_{D}(x)^{1+\beta(p)}} d x<\infty
$$

then $s \equiv 0$ in $D$.

Furthermore, if the absolute value $|s|$ of a subharmonic function $s$ in $D$ satisfies (3) instead of $s$ for some $0<p<(n-1) / n$, then $s \equiv 0$.

Proof. We first quote some properties of $C^{1,1}$-domains which are shown in $[8$, Theorems $2.3,2.4,2.5]$ (see also [6, Lemmas 5, 6]). Let $x_{0}$ be a fixed point in $D$. For $r>0$, we set

$$
\Omega_{r}=\left\{x \in D ; G_{D}\left(x, x_{0}\right)>r\right\} .
$$

Then there are positive numbers $r_{0}$ and $c_{0}=c\left(D, x_{0}\right)$ such that for any $0<r<r_{0}$,

$$
c_{0}^{-1} r \leq \delta_{D}(x) \leqq c_{0} r \quad \text { for any } \quad x \in \partial \Omega_{r} ;
$$

(b) $\Omega_{r}$ is a smooth domain and satisfies

$$
c_{0}^{-1} d x \leq d \omega_{r}^{0} d r \leq c_{0} d x \quad \text { on } \quad D \backslash \Omega_{r_{0}},
$$

where $\omega_{r}^{0}$ is the harmonic measure on $\partial \Omega_{r}$ at $x_{0}$.

Now let $s \geq 0$ be a subharmonic function in $D$ satisfying (3) and suppose $0<p \leq 1$. For $x \in D$, let $B=B\left(x, \delta_{D}(x)\right)$. Then

$$
s(x)^{p} \leq c(n, p) \delta_{D}(x)^{-n} \int_{B} s(y)^{p} d y
$$

This inequality is proved in the same manner as in [1, p. 172]. Remarking $\delta_{D}(y) \leq 2 \delta_{D}(x)$ for $y \in B$, we have

$$
s(x)^{p} \leq c(n, p) \delta_{D}(x)^{(1-n) p} \int_{D} s(y)^{p} \delta_{D}(y)^{-1-\beta(p)} d y
$$

and hence $s^{p-1} \geq c(n, p, s) \delta_{D}^{\beta(p)}$ holds. Therefore by (a) and (b),

$$
\infty>\int_{D \backslash \Omega_{r_{0}}} s(x)^{p} \delta_{D}(x)^{-1-\beta(p)} d x
$$




$$
\begin{aligned}
& \geq c\left(D, x_{0}, p, s\right) \int_{0}^{r_{0}} \int_{\partial \Omega_{r}} s(r \xi)^{p} r^{-1-\beta(p)} d \omega_{r}^{0}(\xi) d r \quad(x=r \xi) \\
& \geq c\left(D, x_{0}, p, s\right) \int_{0}^{r_{0}}\left(\int_{\partial \Omega_{r}} s(r \xi)^{p} d \omega_{r}^{0}(\xi)\right) r^{-1-\beta(p)}\left(\min _{\xi \in \partial_{r}} s(r \xi)^{p-1}\right) d r \\
& \geq c\left(D, x_{0}, p, s\right) s\left(x_{0}\right) \int_{0}^{r_{0}} r^{-1-\beta(p)} r^{\beta(p)} d r,
\end{aligned}
$$

which shows $s\left(x_{0}\right)=0$. Since this holds for all $x_{0} \in D$ we have $s \equiv 0$.

The case $p>1$ follows from the case $p=1$, since $s^{p}$ is also subharmonic.

Next let $s$ be a subharmonic function in $D$ satisfying

$$
\int_{D}|s(x)|^{p} \delta_{D}(x)^{-1-\beta(p)} d x<\infty .
$$

Applying the previous argument to the positive part $s^{+}$of $s$, we see $s^{+} \equiv 0$, because $s^{+}$is also subharmonic. Thus $-s$ is a non-negative superharmonic function in $D$. Suppose $s \neq 0$. Since a $C^{1,1}$-domain is associated with a ball in the sense of [7], by [7, Théorème B] we have $-s \geq c \delta_{D}$ with some $c>0$. However, if $0<p<n /(n-1)$, then $\beta(p) \geq p$, so that we have

$$
\int_{D}|s(x)|^{p} \delta_{D}(x)^{-1-\beta(p)} d x \geq c^{p} \int_{D} \delta_{D}(x)^{p-1-\beta(p)} d x=\infty
$$

which contradicts our assumption. Hence $s \equiv 0$ and the proof is completed.

Before concluding this section, we make two remarks. First, the constant $\beta(p)$ in Theorem 2 can not be replaced by any smaller number.

REMARK 1. Let $B=B(0,1)$ be the unit ball in $R^{n}$ and $p>0$. For any $\varepsilon>0$, there is a non-negative non-zero subharmonic function $s$ on $B$ satisfying

$$
\int_{B} s(x)^{p} \delta_{B}(x)^{\varepsilon-1-\beta(p)} d x<\infty .
$$

In fact, we may consider a constant function in the case $p \geq 1$. In the case $0<p<1$, let $\xi_{0}=(1,0, \cdots, 0) \in \boldsymbol{R}^{n}$. For $0<\theta<\pi / 2$, we choose $r_{\theta}>0$ and $c(\theta)>0$ such that for the truncated circular cone

$$
\Gamma(\theta)=\left\{x \in B ;\left(\xi_{0}-x, \xi_{0}\right)>\left|x-\xi_{0}\right| \cos \theta\right\} \cap B\left(\xi_{0}, r_{\theta}\right)
$$

we have $\delta_{B}(x) \geq c(\theta)\left|x-\xi_{0}\right|$ whenever $x \in \Gamma(\theta)$. Let $h_{\theta}$ be a kernel function at $\xi_{0}$ in $\Gamma(\theta)$ (i.e., $h_{\theta}$ is a positive harmonic function in $\Gamma(\theta)$ vanishing continuously on $\left.\partial \Gamma(\theta) \backslash\left\{\xi_{0}\right\}\right)$. Then

$$
h_{\theta}(x) \leq c(\theta)\left|x-\xi_{0}\right|^{1-n-r(\theta)}
$$

with $\gamma(\theta)>0$, which satisfies $\gamma(\theta) \rightarrow 0$ as $\theta \rightarrow \pi / 2$ (cf. [5, Lemma 3]). Choose $\theta$ such that $p \gamma(\theta)<\varepsilon$. Then the function $s$ in $B$ defined by 


$$
s(x)=\left\{\begin{array}{cl}
h_{\theta}(x) & x \in \Gamma(\theta) \\
0 & x \in B \backslash \Gamma(\theta)
\end{array}\right.
$$

is subharmonic and satisfies

$$
\begin{aligned}
& \int_{B} s(x)^{p} \delta_{B}(x)^{\varepsilon-1-\beta(p)} d x \\
& \quad \leq c(\theta, p) \int_{\Gamma(\theta)}\left|x-\xi_{0}\right|^{(1-n-\gamma(\theta)) p}\left|x-\xi_{0}\right|^{\varepsilon-1-\beta(p)} d x<\infty .
\end{aligned}
$$

In the following remark, let $\beta^{\prime}(p)=\min \{\beta(p), p\}$.

REMARK 2. If a non-negative harmonic function $h$ on a $C^{1,1}$-domain $D$ satisfies

$$
\int_{D} h(x)^{p} \delta_{D}(x)^{-1-\beta^{\prime}(p)} d x<\infty
$$

for some $p>0$, then $h \equiv 0$ holds.

In fact, this follows from Theorem 2 (in the case $p \geq(n-1) / n$ ) and from the argument as in the last part of the proof of Theorem 2 (in the case $0<p$ $<(n-1) / n)$.

Furthermore, by the direct computation, we see that the Poisson kernel of $B(O, 1)$, that is,

$$
h(x)=\frac{1-|x|^{2}}{\left|\xi_{0}-x\right|^{n}} \quad \xi_{0}=(1,0, \cdots, 0)
$$

is an example proving the sharpness of the constant $\beta^{\prime}(p)$ for $p \leq 1$ (for $p>1$, we may consider the constant function as before).

\section{$\S 4$. Non-tangential positiveness of harmonic functions}

In this section $D$ is assume to be a Lipschitz domain in $\boldsymbol{R}^{n}$. Let $\xi \in \partial D$. By a cone at $\xi$ we mean an open truncated circular cone with vertex at $\xi$. We say that a line segment $T$ in $D$ is a non-tangential (abbreviated to n.t.) segment at $\xi$ if there is a cone $\Gamma$ at $\xi$ in $D$ such that its axis of symmetry is $T$. A cone $\Gamma$ at $\xi$ is also called an n.t. cone in $D$ if there is a cone $\Gamma^{\prime}$ such that $\bar{\Gamma} \backslash\{\xi\} \subset \Gamma^{\prime} \subset D$.

Let $h$ be a non-negative harmonic function in $D$. We say that $h$ is nontangentially positive at $\xi \in \partial D$, if there is an n.t. segment $T$ at $\xi$ such that

$$
\liminf _{\substack{x \rightarrow \xi \\ x \in T}} h(x)>0 .
$$


Then by the Harnack inequality, $h$ has the positive infimum on any n.t. cone at $\xi$. Hence this positivity is independent of the choice of n.t. segment at $\xi$, since $D$ is a Lipschitz domain.

We denote by $P_{D}(x, \xi)$ the Martin (Poisson) kernel on $D \times \partial D$ (normalized at some point $x_{0} \in D$ ) (cf. [3]). We have the following result.

THEOREM 3. Let $D$ be a bounded Lipschitz domain in $\boldsymbol{R}^{n}$ with the uniform exterior sphere property. Let $h$ be a non-negative harmonic function in $D$ and let $\mu_{h}$ be the non-negative Borel measure on $\partial D$ which represents $h$, that is,

$$
h=\int_{\partial D} P_{D}(\cdot, \xi) d \mu_{h}(\xi)
$$

Then $h$ is non-tangentially positive $\mu_{h}$-a.e., namely if we put $A_{h}=\{\xi \in \partial D ; h$ is non-tangentially positive at $\xi\}$, then $A_{h}$ is Borel measurable and $\mu_{h}\left(\partial D \backslash A_{h}\right)=0$.

For the proof, we prepare the following lemma.

Lemma 1. Let $D$ and $\Omega$ be two Lipschitz domains with $x_{0} \in D \subset \Omega$. Suppose that $\xi \in \partial D \cap \partial \Omega$ and let $T$ be an n.t. segment at $\xi$ in $D$. If $u=P_{D}(\cdot, \xi)$ and $v=P_{o}(\cdot, \xi)$ satisfy $u\left(x_{0}\right)=v\left(x_{0}\right)=1$, then

$$
\liminf _{\substack{x \rightarrow \xi \\ x \in T}} \frac{u(x)}{v(x)} \geq 1
$$

Furthermore, for any n.t. cone $\Gamma$ at $\xi$ in $D$, we have $u(x) \geq c(\Gamma) v(x)$ for $x \in \Gamma$ with some constant $c(\Gamma)>0$.

Proof. We follow the argument in the proof of [3, Lemma (3.7)]. Suppose that (4) does not hold. Then there exist a constant $\varepsilon>0$ and a sequence $\left\{x_{n}\right\}$ in $T$ such that $\lim _{n \rightarrow \infty} x_{n}=\xi$ and $u\left(x_{n}\right)<(1-2 \varepsilon) v\left(x_{n}\right)$ for $n \geq 1$. By the Harnack inequality we can take $r_{0}>0$ satisfying $B:=\bigcup_{u=1}^{\infty} B\left(x_{n}, r_{0}\left|x_{n}-\xi\right|\right) \subset D$ and

$$
u(x)<(1-\varepsilon) v(x) \text { for } x \in B .
$$

From [3, Lemma (3.6)] it follows that

$$
\begin{aligned}
& 1=u\left(x_{0}\right)=R_{u}^{B, D}\left(x_{0}\right) \\
& :=\inf s\left(x_{0}\right) ;\{s \geq 0 \text { superharmonic in } D \text { with } s \geq u \text { on } B\} \\
& \quad \leq \inf \left\{\tilde{s}\left(x_{0}\right) ; \tilde{s} \geq 0 \text { superharmonic in } \Omega \text { with } \tilde{s} \geq(1-\varepsilon) v \text { on } B\right\} \\
& :=R_{(1-\varepsilon) v}^{B, \Omega}\left(x_{0}\right) \leq(1-\varepsilon) v\left(x_{0}\right)=1-\varepsilon .
\end{aligned}
$$

This contradiction proves (4). The latter assertion of the lemma follows immediately by the Harnack inequality. 
Proof of Theorem 3. First we shall show that $A_{h}=\varnothing$ implies $h \equiv 0$. By the uniform exterior sphere property of $D$, there exists $r_{0}>0$ such that for each $\xi \in \partial D$ there corresponds a point $\xi^{\prime}$ such that $\left|\xi-\xi^{\prime}\right|=r_{0}$ and $B\left(\xi^{\prime}, r_{0}\right) \subset \bar{D}^{c}$. Take $r_{1}>0$ sufficiently large so that

$$
\Omega_{\xi}:=B\left(\xi^{\prime}, r_{1}\right) \backslash \overline{B\left(\xi^{\prime}, r_{0}\right)}
$$

contains $D$ for any $\xi \in \partial D$.

Let $\eta$ be an arbitrary fixed point of $\partial D$. Since $D$ is a Lipschitz domain, there exist an n.t. segment $T_{0}$ at $\eta$ in $D$ and a constant $r_{2}>0$ such that for any $\xi \in \partial D \cap B\left(\eta, r_{2}\right)$,

$$
T_{\xi}=\left\{x-\eta+\xi ; x \in T_{0}\right\}
$$

is an n.t. segment at $\xi$. Denote $\xi(r)=r e_{0}+\xi(\subset D)$, where $e_{0}$ is the unit vector parallel to $T_{0}$. Since $\left\{\Omega_{\varepsilon}\right\}$ are mutually congruent $C^{\infty}$-domains, we see ([8, Theorem 2.3])

$$
P_{Q_{\xi}}(\xi(r), \xi) \geq c(D, \eta) r^{1-n}
$$

for any $0<r<r_{2}$ and for any $\xi \in \partial D \cap B(\eta, r)\left(P_{\Omega_{\xi}}\right.$ is also normalized at $x_{0} \in D$ $\left.\subset \Omega_{\xi}\right)$. Hence by Lemma 1 and by the Harnack inequality, we have

$$
P_{D}(\eta(r), \xi) \geq c(D, \eta) P_{D}(\xi(r), \xi) \geq c(D, \eta) r^{1-n}
$$

for any $\xi \in \partial D \cap B(\eta, r / M)$, where $M$ is a positive constant depending only on D. Therefore

$$
\begin{aligned}
h(\eta(r)) & \geq \int_{\partial D \cap B(n, r / M)} P_{D}(\eta(r), \xi) d \mu_{n}(\xi) \\
& \geq c(D, \eta) r^{1-n} \mu_{h}(B(\eta, r / M)) .
\end{aligned}
$$

Denoting by $\sigma$ the surface measure on $\partial D$, we have $\sigma(B(\eta, r)) \geq c(D) r^{n-1}$ and hence

$$
h(\eta(r)) \sigma(B(\eta, r / 5 M)) \geq c(D, \eta) \mu_{h}(B(\eta, r / M))
$$

Let $\varepsilon>0$. By our assumption on $h, \liminf _{r \rightarrow 0} h(\eta(r))=0$ and hence there is $r_{h}>0$ satisfying $\varepsilon \sigma\left(B\left(\eta, r_{n} / 5\right)\right) \geq \mu_{h}\left(B\left(\eta, r_{\eta}\right)\right)$. Since $\left\{B\left(\eta, r_{\eta} / 5\right) ; \eta \in \partial D\right\}$ is an open covering of $\partial D$, we can choose finite points $\eta_{1}, \eta_{2}, \cdots, \eta_{m}$ in $\partial D$ such that

$$
\partial D=\bigcup_{i=j}^{m} B\left(\eta_{i}, r_{\eta_{i}}\right)
$$

and $\left\{B\left(\eta_{i}, r_{\eta_{i}} / 5\right)\right\}_{i=1}^{m}$ are mutually disjoint. Thus we have

$$
\mu_{h}(\partial D) \leq\left(\sum_{i=1}^{m} \mu_{h} B\left(\eta_{i}, r_{h_{i}}\right)\right) \leq \varepsilon \sum_{i=1}^{m} \sigma\left(B\left(\eta_{i}, r_{\eta_{i}} / 5\right)\right) \leq \varepsilon \sigma(\partial D) .
$$


Since $\varepsilon>0$ is arbitrary, $\mu_{h}=0$ and hence $h \equiv 0$.

Next we consider the case $A_{h} \neq \varnothing$. We see that $A_{h}$ is a Borel subset of $\partial D$, since $h$ is a continuous function in $D$ and since we can choose n.t. segments $T_{\xi}$ which vary continuously in $\xi \in \partial D$ (note the argument in the second paragraph of this proof). Suppose that $\mu_{h}\left(\partial D \backslash A_{h}\right)>0$. Since $\mu_{h}$ is regular, there exists an open set $U$ in $\partial D$ such that $U \supset A_{h}$ and $\mu_{h}(\partial D \backslash U)>0$. Letting $\tilde{\mu}$ be the restriction of $\mu_{h}$ to $\partial D \backslash U$ and letting

$$
\tilde{h}=\int_{\partial D} P_{D}(\cdot, \xi) d \tilde{\mu}(\xi),
$$

we see $A_{\tilde{h}} \subset A_{h} \subset U$ and $A_{\tilde{h}} \neq \varnothing$, because $\tilde{h} \neq 0$. On the other hand, since $\operatorname{supp}(\tilde{\mu}) \subset \partial D \backslash U, \tilde{h}$ vanishes continuously on $U$, which implies $A_{\tilde{h}} \subset \partial D \backslash U$. This contradiction proves $\mu_{h}\left(\partial D \backslash A_{h}\right)=0$ and the proof is completed.

\section{§5. Proof of Theorem A}

The case $0<p \leq(n-2) /(n-1)$ : The result follows from Theorem 1 , since $h^{p}$ is superharmonic in $D$.

The case $(n-2) /(n-1)<p \leq 1$ : We denote by $\Delta$ the Martin boundary of $D$ and by $K(x, \lambda)$ the Martin kernel on $D \times \Delta$. The set of all minimal boundary points of $\Delta$ is denoted by $\Delta_{1}$. Then there exists a non-negative Borel measure $\mu$ on $\Delta_{1}$ such that

$$
h=\int_{\Delta_{1}} K(\cdot, \lambda) d \mu(\lambda)
$$

(see [2, Ch. 12]). We may assume $\mu\left(\Delta_{1}\right) \leq 1$.

Let $\lambda \in \Delta_{1}$. Since $K(\cdot, \lambda)$ is minimal we see by the assumption on $\partial D$ that there is a point $\xi_{\lambda}$ in $\partial D$ such that $K(\cdot, \lambda)$ vanishes continuously on $\partial D \backslash\left\{\xi_{\lambda}\right\}$ (see [4]). Take two balls $B_{\lambda}, B_{\lambda}^{\prime}$ such that

$$
B_{\lambda} \subset \bar{D}^{c}, \quad \bar{B}_{\lambda} \cap \partial D=\left\{\xi_{\lambda}\right\} \text { and } \overline{D \cup B_{\lambda}} \subset B_{\lambda}^{\prime} .
$$

Let $D_{\lambda}=B_{\lambda}^{\prime} \backslash \bar{B}_{\lambda}$ and define a function $s_{\lambda}$ on $D_{\lambda}$ by

$$
s_{\lambda}(x)=\left\{\begin{array}{cl}
K(x, \lambda) & x \in D \\
0 & x \in D_{\lambda} \backslash D,
\end{array}\right.
$$

then $s_{\lambda}$ is a positive subharmonic function on a $C^{1,1}$-domain $D_{\lambda}$. Noting that $\delta_{D_{\lambda}}(x) \geq \delta_{D}(x)$ for $x \in D$ and $\alpha(p)=\beta(p)$, we have

$$
\int_{D} K(x, \lambda)^{p} \delta_{D}(x)^{-1-\alpha(p)} d x \geq \int_{D_{\lambda}} s_{\lambda}(x)^{p} \delta_{D_{\lambda}}(x)^{-1-\beta(p)} d x=\infty
$$

by Theorem 2. By the Hölder inequality and the Fubini theorem, 


$$
\begin{aligned}
\int_{D} h(x)^{p} \delta_{D}(x)^{-1-\alpha(p)} d x & \geq \int_{D} \delta_{D}(x)^{-1-\alpha(p)}\left(\int_{\Delta_{1}} K(x, \lambda)^{p} d \mu(\lambda)\right) d x \\
& =\int_{\Delta_{1}} \int_{D} K(x, \lambda)^{p} \delta_{D}(x)^{-1-\alpha(p)} d x d \mu(\lambda),
\end{aligned}
$$

so that (1) implies $\mu=0$ and consequently $h \equiv 0$.

The case $p>1$ : As before, we represent $h$ as

$$
h=\int_{\Delta_{1}} K(\cdot, \lambda) d \mu(\lambda)
$$

For $m \geq 1$, put $F_{m}=\left\{x \in \boldsymbol{R}^{n} ; \operatorname{dist}(x, F) \geq 1 / m\right\}$, where $F$ is the countable closed set in Theorem A. We take a Lipschitz domain $D_{m}$ with the uniform exterior property such that $D \cap F_{2 m} \subset D_{m} \subset D$. Then in $D_{m}$ we have $h \int_{\partial D_{m}} P_{m}(\cdot, \xi) d \mu_{m}(\xi)$ with some positive measure $\mu_{m}$ on $\partial D_{m}$, where $P_{m}=P_{D_{m}}$. Now put

$$
h_{m}(x)=\int_{\partial D_{m} \cap F_{m}} P_{m}(x, \xi) d \mu_{m}(\xi) .
$$

We shall show that $h_{m} \equiv 0$ in $D_{m}$ for every $m \geq 1$. By Theorem 3 , we have $\mu_{m}\left(\partial D_{m} \cap F_{m} \backslash A_{m}\right)=0$, where $A_{m}=\left\{\xi \in \partial D_{m} ; h_{m}\right.$ is non-tangentially positive at $\xi\}$. For $\xi \in A_{m}$, let $\Gamma_{\xi}$ be an n.t. cone at $\xi$ in $D_{m}$. Then $h_{m} \geq c_{\xi}$ on $\Gamma_{\xi}$ with some constant $c_{\xi}>0$. On the other hand, by the exterior sphere property of $D_{m}, P_{m}(x, \xi) \geq c\left(D_{m}, \Gamma_{\xi}\right)|x-\xi|^{1-n}$ for $x \in \Gamma_{\xi}$ (see Lemma 1) and hence

$$
\int_{\Gamma \xi} P_{m}(x, \xi) \delta_{D}(x)^{-1} d x \geq c\left(D_{m}, \Gamma_{\xi}\right) \int_{\Gamma_{\xi}}|x-\xi|^{-n} d x=\infty
$$

Therefore

$$
\begin{aligned}
\int_{D} h(x)^{p} \delta_{D}(x)^{-1} d x & \geq \int_{D_{m}} h_{m}(x)^{p} \delta_{D}(x)^{-1} d x \\
& =\int_{\partial_{D_{m}}} \int_{D_{m}} P_{m}(x, \xi) \delta_{D}(x)^{-1}\left(h_{m}(x)^{p-1}\right) d x d \mu_{m}(\xi) \\
& \geq \int_{A_{m}}\left(\int_{\Gamma_{m}} P_{m}(x, \xi) \delta_{D}(x)^{-1} c_{\xi}^{p-1} d x\right) d \mu_{m}(\xi)
\end{aligned}
$$

so that (1) implies $\mu_{m}\left(\partial D_{m} \cap F_{m}\right)=\mu_{m}\left(A_{m}\right)=0$, and hence $h_{m} \equiv 0$. Hence $h$ vanishes continuously on $\partial D \cap F_{m}^{c}$ for all $m \geq 1$, that is, $h$ has boundary value zero on $\partial D \backslash F$.

For $\xi \in F$, we put

$$
M_{\xi}=\left\{\lambda \in \Delta_{1} ; K(\cdot, \lambda) \text { vanishes continuously on } \partial D \backslash\{\xi\}\right\} .
$$

Then $M_{\xi}$ is closed in $\Delta_{1}$ and

$$
\mu\left(\Delta_{1}\right)=\sum_{\xi \in F} \mu\left(M_{\xi}\right) .
$$


We take two balls $B_{1}$ and $B_{2}$ such that $B_{1} \subset D^{c}, \bar{B}_{1} \cap \partial D=\{\xi\}$ and $B_{2} \supset \overline{D \cup B}_{1}$. Define a function $s$ in $\tilde{D}=B_{2} \backslash \bar{B}_{1}$ by

$$
s(x)= \begin{cases}\left(\int_{M_{\xi}} K(\cdot, \lambda) d_{\mu}(\lambda)\right)^{p} & x \in D \\ 0 & x \in \tilde{D} \backslash D,\end{cases}
$$

then $s$ is a non-negative subharmonic function in a $C^{1,1}$-domain $\tilde{D}$. Since

$$
\int_{\tilde{D}} s(x) \delta_{\tilde{D}}(x)^{-1} d x \leq \int_{D} h(x)^{p} \delta_{D}(x)^{-1} d x<\infty,
$$

Theorem 2 shows $s \equiv 0$ or $\mu\left(M_{\xi}\right)=0$. Since this holds for every $\xi \in F$ we have $\mu=0$ and consequently $h \equiv 0$ in $D$. The proof is completed.

Added IN Proof. After submitted this paper, Prof. H. Aikawa pointed out that the assertion of Theorem 3 was given in a paper of J. M. Wu [Ann. Inst. Fourier 28, 4 (1978) 147-167]. She proved this result by comparing representing measure and harmonic measure instead of surface measure.

\title{
References
}

[1] C. Fefferman and E. M. Stein, $H^{p}$ spaces of several variables, Acta Math., 129 (1972), 137-193.

[ 2 ] L. L. Helms, Introduction to Potential Theory, Wiley-Interscience, New York, 1969.

[3] R. A. Hunt and R. L. Wheeden, Positive harmonic functions on Lipschitz domains, Trans. Amer. Math Soc., 147 (1970), 507-527.

[4] P. J. Kostense, On the behaviour of minimal positive harmonic functions at the boundary of their domain, Nederl. Akad. Wetensch. Proc. Ser. A, 80, Indag. Math. (1977), 23-28.

[5] F-Y. Maeda and N. Suzuki, The integrability of superharmonic functions on Lipschitz domains, Bull. London Math. Soc., 21 (1989), 270-278.

[6] N. Suzuki, Martin boundary for $\Delta-P$, Hiroshima Math. J., 14 (1984), 67-74.

[7] N. Suzuki, L'allure à la frontière des fonctions L-harmoniques positives dans un domaine, C. R. Acad. Sci. Paris Sér. I, 303 (1986), 621-624.

[8] K.-O. Widman, Inequalities for the Green function and boundary continuity of the gradient of solutions of elliptic differential equations, Math. Scand., 21 (1967), 17-37.

\author{
Department of Mathematics \\ FACULTY OF SCIENCE \\ HIROSHIMA UNIVERSITY \\ CURRENT ADDRESS: \\ Department of Mathematics \\ College of General Education \\ NAGOYA UNIVERSITY
}

